\title{
Slow-Light Optical Bullets in Arrays of Nonlinear Bragg-Grating Waveguides
}

\author{
Andrey A. Sukhorukov and Yuri S. Kivshar \\ Nonlinear Physics Centre and Centre for Ultrahigh-bandwidth Devices for Optical Systems (CUDOS), \\ Research School of Physical Sciences and Engineering, Australian National University, ACT 0200 Canberra, Australia*
}

(Received 4 July 2006; published 4 December 2006)

\begin{abstract}
We demonstrate that propagation direction and velocity of optical pulses can be controlled independently in the structures with multiscale modulation of the refractive index in transverse and longitudinal directions. We reveal that, in arrays of waveguides with phase-shifted Bragg gratings, the refraction angle does not depend on the speed of light, allowing for efficient spatial steering of slow light. In this system, both spatial diffraction and temporal dispersion can be designed independently, and we identify the possibility for self-collimation of slow light when spatial diffraction is suppressed for certain propagation directions. We also show that broadening of pulses in space and time can be eliminated in nonlinear media, supporting the formation of slow-light optical bullets that remain localized irrespective of propagation direction.
\end{abstract}

DOI: 10.1103/PhysRevLett.97.233901

PACS numbers: 42.79.Gn, 42.65.Tg, 42.70.Qs, 42.79.Dj

Photonic structures with a periodic modulation of the optical refractive index open novel possibilities for controlling the flow of light [1]. Transmission of optical waves is suppressed for a range of frequencies associated with photonic band gaps, which appear due to the Bragg scattering. The energy flow in propagating waves and, accordingly, their group velocity are gradually reduced as the gap edge is approached. Slowing down of light pulses was observed in fibers with Bragg gratings [2]. Most recently, reduction of the speed of light by a factor approaching 1000 was registered in coupled-defect waveguides in photonic crystals (see, e.g., Refs. [3-5]). The speed of light can be adjusted dynamically by changing the refractive index and shifting the band gaps. Such tunability is achieved by a variety of methods, including recently demonstrated thermal control [3]. The group velocity and pulse delay can be tuned all-optically in nonlinear photonic structures, where the photonic band gaps are shifted by a pump wave or by the pulse itself [2] even at moderate powers due to enhanced efficiency of nonlinear interactions in the slow-light regime [6]. A very important advantage of this approach is that the nonlinearity can lead to pulse self-trapping and suppress its broadening due to dispersion [2], overcoming the limitations of linear devices [7].

Efficient beam steering was demonstrated in photonic structures, where beam refraction and diffraction can be engineered. However, simultaneous control of pulse speed and its propagation direction is a challenging problem. For example, special design of waveguide bends is required for slow-light pulses [8]. Dynamical steering can be realized in periodic structures, where the propagation direction is not fixed by a prefabricated waveguide [9]. In such structures, the speed of light may be controlled through the band-gap shift similar to fixed waveguides. However, for previously studied symmetric $2 \mathrm{D}$ or $3 \mathrm{D}$ defect-free photonic-crystal structures, the beam refraction is strongly modified as the group velocity is reduced due to reshaping and shrinkage of the isofrequency dispersion curves near the gap edge [1].

In this Letter, we demonstrate that the propagation direction and velocity of optical pulses can be independently controlled in photonic structures with multiple-scale modulations of the refractive index in transverse and longitudinal directions. We show that this regime can be realized in arrays of coupled waveguides with a transverse size of the order of micrometers. It was demonstrated that such modulation in the transverse spatial dimension fundamentally affects the beam refraction and enables tunable beam steering in nonlinear structures [10]. We reveal that, by introducing refractive index modulation along the waveguides satisfying the Bragg resonance condition at the operating frequency, it becomes possible to simultaneously slow down light pulses and perform their spatial steering. Additionally, we show how to engineer independently the strength of diffraction and dispersion in the slow-light regime, providing the optimal conditions for the nonlinear control of spatiotemporal pulse dynamics. In particular, we predict and demonstrate numerically the formation of strongly localized slow-light optical bullets in such structures, which overcome the issue of pulse broadening in linear systems. Suggested structures can be fabricated, for example, in AlGaAs samples, where the gratings and the waveguides can be defined simultaneously by use of a onestep electron-beam-based approach [11].

We consider propagation of pulses whose frequency is tuned in the vicinity of Bragg resonance. Then the pulse dynamics can be modeled by a set of coupled-mode nonlinear equations [12] for the slowly varying envelopes of the forward $\left(u_{n}\right)$ and backward $\left(v_{n}\right)$ propagating fields in each of the $n$th waveguides. In the normalized form, these equations can be written as follows: 


$$
\begin{aligned}
i \frac{\partial u_{n}}{\partial t}+i \frac{\partial u_{n}}{\partial z}+ & C\left(u_{n-1}+u_{n+1}\right) \\
& +\rho_{n} v_{n}+\gamma\left(\left|u_{n}\right|^{2}+2\left|v_{n}\right|^{2}\right) u_{n}=0, \\
i \frac{\partial v_{n}}{\partial t}-i \frac{\partial v_{n}}{\partial z}+ & C\left(v_{n-1}+v_{n+1}\right) \\
& +\rho_{n}^{*} u_{n}+\gamma\left(\left|v_{n}\right|^{2}+2\left|u_{n}\right|^{2}\right) v_{n}=0,
\end{aligned}
$$

where $t$ and $z$ are the dimensionless time and propagation distance normalized to $t_{s}$ and $z_{s}$, respectively, $C$ is the coupling coefficient for the modes of the neighboring waveguides, $\rho_{n}$ characterizes the scattering strength of the Bragg grating, $\gamma$ is the nonlinear coefficient, and the group velocity far from the Bragg resonance is normalized to unity. For $\left|\rho_{n}\right|=1$, the scaling coefficients are $t_{s}=$ $\lambda_{0}^{2} /\left(\pi c \Delta \lambda_{0}\right)$ and $z_{s}=t_{s} c / n_{0}$, where $c$ is the speed of light in vacuum, $\lambda_{0}$ is the wavelength in vacuum, $\Delta \lambda_{0}$ is the width of the Bragg resonance for an individual waveguide, and $n_{0}$ is the average refractive index.

We reveal that both diffraction and dispersion can be precisely tailored by introducing a phase shift between the otherwise equivalent waveguide gratings, as illustrated in Figs. 1(a) and 1(b). Only two- and three-waveguide nonlinear couplers with in-phase gratings were analyzed before [13-15]. Here we consider the effect of a linear phase shift of the gratings across the array characterized by the scattering coefficients $\rho_{n}=\rho \exp (-i \varphi n)$. With no loss of generality, we can take $\rho$ to be real and positive. Then the wave propagation in the linear regime (at small intensities) can be fully defined through the properties of FloquetBloch eigenmodes, $u_{n}=u_{0} \exp [i K n+i \beta z-i \omega t], v_{n}=$ $v_{0} \exp [i(K+\varphi) n+i \beta z-i \omega t]$, where $K$ and $\beta$ are the transverse and longitudinal components, respectively, of the Bloch wave vector. After substituting these expressions into the linearized equations (1) (with $\gamma=0$ ), we obtain the following relations: $u_{0}[\omega-\beta+2 C \cos (K)]+v_{0} \rho=$ $0, u_{0} \rho+v_{0}[\omega+\beta+2 C \cos (K+\varphi)]=0$. These eigenmode equations define the dispersion properties of the Bloch waves $\omega(K, \beta)$, which determine the key features of the wave spectrum. First, the gaps may appear for a certain frequency range, where the propagating waves with real $K$ and $\beta$ are absent. We notice that quasi-2D spectral gaps can indeed appear for the modes localized in the highindex waveguides, i.e., below the light line of the substrate. Second, the spatial beam refraction and diffraction are defined by the shape of the corresponding isofrequency contours. The propagation angle is defined by the value of $\alpha(\omega, K)=-\partial \beta / \partial K$, and the effective diffraction experienced by the beam depends on the curvature, $D(\omega, K)=$ $-\partial^{2} \beta / \partial K^{2}$. Below, we analyze how these fundamental characteristics can be controlled by selecting the phase shift $\varphi$ between the Bragg gratings.

For the in-phase gratings [i.e., when $\varphi=0$; see Fig. 1(a)], the dispersion relation becomes $\omega(K, \beta)=$ $-2 C \cos (K) \pm\left(\rho^{2}+\beta^{2}\right)^{1 / 2}$. It follows that the 2D gap appears only when the waveguide coupling is weak, $C<$ $\rho / 2$. The regime of large coupling corresponds to the con-
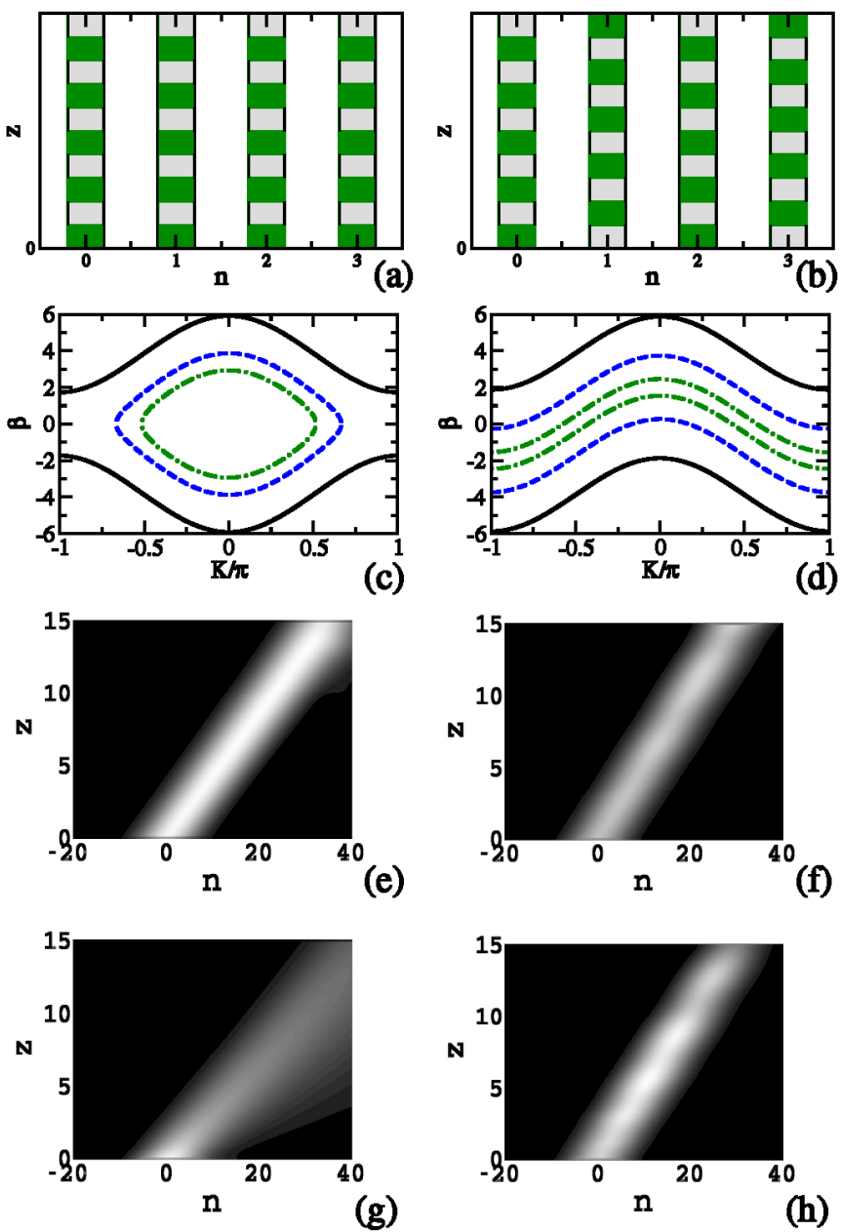

FIG. 1 (color online). (a),(b) Schematic of a waveguide array with (a) in-phase $(\varphi=0)$ and (b) phase-shifted $(\varphi=\pi)$ Bragg gratings; (c),(d) corresponding isofrequency contours for different detuning from the gap edge: $\omega=4$ (solid line), $\omega=2$ (dashed line), and $\omega=1.1$ (dashed-dotted line). (e)-(h) Beam refraction with incident angle corresponding to $K_{b}=\pi / 2$ for (e),(f) $\omega=2$ and (g),(h) $\omega=1$.1. For all the plots, $\rho=1$ and $C=1$.

tinuum limit, and our conclusion on the absence of 2D gap in this case agrees with earlier predictions that only quasilocalized states with a large spatial extent may exist in a slab waveguide with a 1D Bragg grating [16]. We also note that the shape of the isofrequency contours is strongly frequency-dependent as the transmission band edge is approached; see Fig. 1(c). This happens because the position of the one-dimensional frequency gap depends on the propagation direction. Since the direction of wave propagation is defined by normal to the isofrequency curves, the beam refraction is strongly modified as it bends towards the horizontal direction for frequency tuning closer to the gap edge [cf. Figs. 1(e) and 1(g)]. Additionally, the beam is strongly broadened due to enhanced diffraction in the slow-light regime, which drastically reduces the spatial resolution.

We find that the nature of wave dispersion is fundamentally altered for the waveguide structure with out-of-phase 
shift of the neighboring gratings [i.e., when $\varphi=\pi$; see Fig. 1(b)]. The corresponding dispersion relation has a different form, $\omega(K, \beta)= \pm\left\{\rho^{2}+[\beta-2 C \cos (K)]^{2}\right\}^{1 / 2}$. Moreover, for any propagation angle defined by the transverse Bloch wave vector component $K$, the width and position of the one-dimensional frequency gap remains the same, $|\omega|<\rho$. This unusual property leads to remarkable spectral features. First, the 2D (quasi)gap is always present in the spectrum irrespective of the grating strength $(\rho)$ and coupling between the waveguides $(C)$. Second, the shape of isofrequency contours does not depend on frequency in the transmission band; see Fig. 1(d). This means that the beam refraction and diffraction remain the same even for slow light when the band edge is approached; cf. Figs. 1(f) and 1(h). These examples illustrate the effect of beam selfcollimation, when diffraction vanishes for the chosen incident angle. Most remarkably, the self-collimation behavior is preserved for the slow-light regime as well.

The unique features of linear spectrum in arrays with phase-shifted gratings suggest that these structures provide optimal conditions for a nonlinear control of the pulse dynamics. In particular, since the 2D gap appears for any values of the grating strength and waveguide coupling, it is possible to choose these parameters independently in order to balance the rates of dispersion and diffraction. This allows for simultaneous compensation of the pulse broadening in space and time and the formation of light bullets [17-19] through the nonlinear self-trapping effect. Indeed, we find numerically localized solutions of Eqs. (1) for stationary and moving light bullets of the form of solitary waves $\{u, v\}_{n}(z, t)=\{\tilde{u}, \tilde{v}\}_{n}(z-V t) \exp (-i \omega t)$, where $V$ is the propagation velocity. We confirm that localization is possible in the cases of both positive $(\gamma=+1)$ and negative $(\gamma=-1)$ nonlinear response, since anomalous or normal dispersion regimes can be accessed on either edge of the photonic band gap. The soliton solutions for different signs of $\gamma$ can be mapped by changing $\omega \rightarrow-\omega$ and making a corresponding transformation of pulse profiles. In Fig. 2, we show the characteristics of immobile solitons such as energy and width vs the frequency detuning inside the band gap for self-focusing nonlinearity. These solitons have a well-pronounced $X$ shape near the upper edge of the gap, becoming more localized inside the gap as the pulse energy is increased. We notice, however, that these are fully localized states that should not be confused with the so-called $\mathbf{X}$ waves [20], which remain quasilocalized only over a finite propagation distance. The gap solitons can also propagate along the waveguides, and we present the characteristics of moving solitons in Fig. 3. These slow-light bullets become more extended as the propagation velocity is increased.

Finally, we study spatial steering of pulses across the array. In the linear regime, the pulse broadens in both the transverse and longitudinal directions [Figs. 4(a)-4(d)]. Nonlinear self-action results in the pulse self-trapping in both space and time. In Figs. 4(e)-4(h), the velocity of the
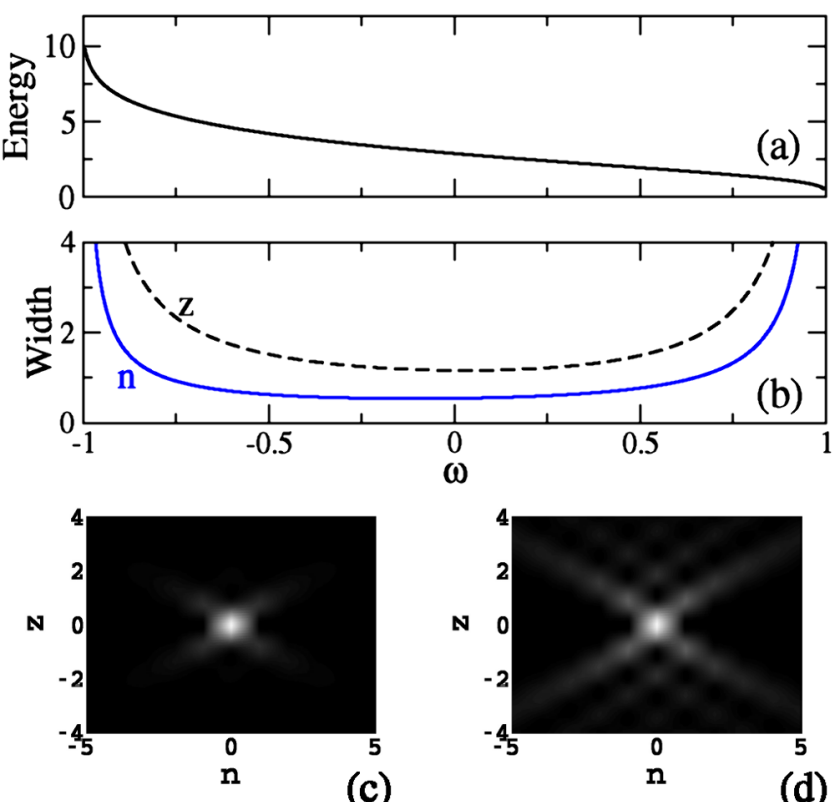

FIG. 2 (color online). Family of the stationary (zero-velocity) light bullets characterized by (a) energy and (b) width along the transverse (solid curve) and longitudinal (dashed curve) directions vs the frequency tuning inside the spectral gap. (c)(d) Intensity profiles of slow-light optical bullets for (c) $\omega=0.8$ and (d) $\omega=0.995$; brighter shading marks higher intensity.

generated light bullet is $30 \%$ of the speed of light in the absence of the Bragg grating, and smaller velocities can be accessed as well by controlling the central frequency and bandwidth of the input pulse. The profile of the self-
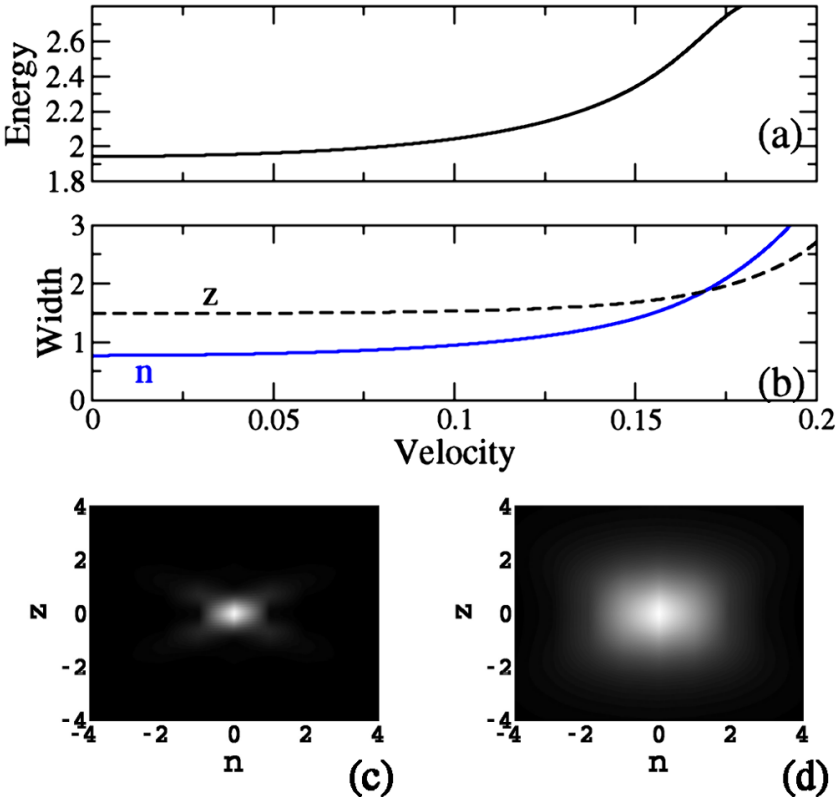

FIG. 3 (color online). Moving slow-light optical bullets with the frequency detuning $\omega=0.5$. Notations are the same as in Fig. 2, and the velocity is normalized to the speed of light away from the Bragg resonance. Profiles are shown for the velocities (c) $V=0.1$ and (d) $V=0.2$. 

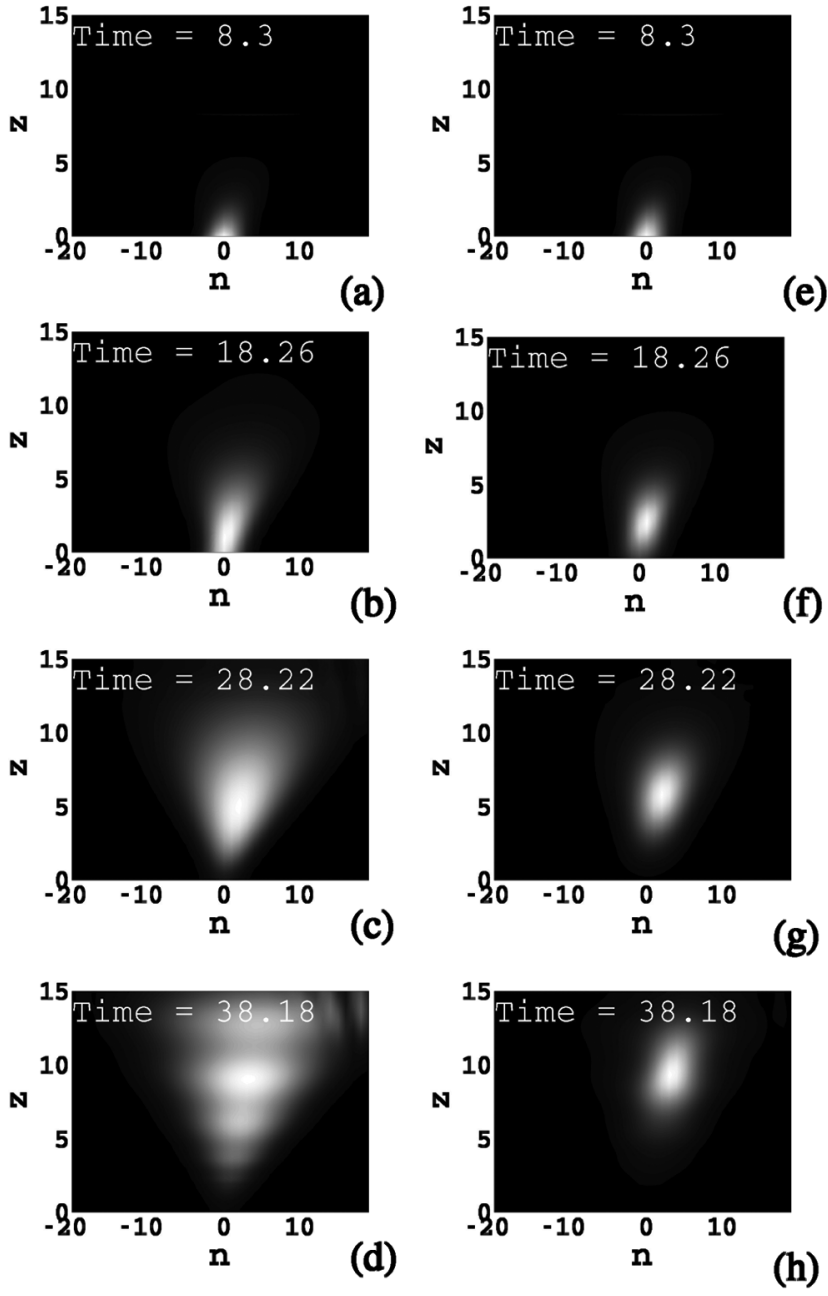

FIG. 4. Snapshots of field intensities for an optical pulse propagating in a waveguide array structure show in Fig. 1(b): (a) -(d) Linear broadening due to spatial diffraction and temporal dispersion; (e)-(h) nonlinear self-trapping in space and time and the formation of an optical bullet. The input pulse has a Gaussian profile with FWHM $\Delta t \simeq 13$.

trapped state [Fig. 4(h)] closely resembles the shape of the soliton solution in Fig. 3(d). Taking the characteristic experimental values for a single Bragg-grating waveguide fabricated in AlGaAs [11] $\Delta \lambda_{0}=0.2 \mathrm{~nm}$ and $\lambda_{0}=$ $1550 \mathrm{~nm}$, we have $t_{s} \simeq 12.8 \mathrm{ps}$ and $z_{s} \simeq 1.8 \mathrm{~mm}$. Accordingly, simulations in Fig. 4 correspond to experimentally feasible conditions of the input pulse duration $170 \mathrm{ps}$ and device length $27 \mathrm{~mm}$. Control of shorter pulses may be realized in deeper gratings with larger bandwidth.

In conclusion, we have revealed that both spatial diffraction and temporal dispersion can be engineered independently near the edge of the photonic band gap in the waveguide arrays with phase-shifted Bragg gratings. We have shown that such structures possess quasi-twodimensional photonic band gaps and provide optimal conditions for self-localization of pulses in nonlinear media. We have demonstrated that these waveguide arrays can be employed for shaping and control of optical pulses simul- taneously in space and time and allow the formation of optical bullets propagating with slow group velocities. Such slow-light optical bullets offer novel possibilities for alloptical switching, steering, and control of short pulses.

We thank M. de Sterke and B. Eggleton for useful discussions. This work has been supported by the Australian Research Council.

*Electronic address: http://www.rsphysse.anu.edu.au/ nonlinear

[1] J. D. Joannopoulos, R. D. Meade, and J. N. Winn, Photonic Crystals: Molding the Flow of Light (Princeton University, Princeton, 1995).

[2] B. J. Eggleton, C. M. de Sterke, and R. E. Slusher, J. Opt. Soc. Am. B 16, 587 (1999); J. T. Mok, C. M. de Sterke, I. C. M. Littler, and B. J. Eggleton, Nature Phys. 2, 775 (2006).

[3] Y. A. Vlasov, M. O'Boyle, H. F. Hamann, and S. J. McNab, Nature (London) 438, 65 (2005).

[4] H. Gersen, T. J. Karle, R. J. P. Engelen, W. Bogaerts, J. P. Korterik, N. F. van Hulst, T. F. Krauss, and L. Kuipers, Phys. Rev. Lett. 94, 073903 (2005).

[5] R.S. Jacobsen, A. V. Lavrinenko, L. H. Frandsen, C. Peucheret, B. Zsigri, G. Moulin, J. Fage Pedersen, and P. I. Borel, Opt. Express 13, 7861 (2005).

[6] M. Soljacic, S. G. Johnson, S. H. Fan, M. Ibanescu, E. Ippen, and J. D. Joannopoulos, J. Opt. Soc. Am. B 19, 2052 (2002).

[7] R. J. P. Engelen, Y. Sugimoto, Y. Watanabe, J. P. Korterik, N. Ikeda, N. F. van Hulst, K. Asakawa, and L. Kuipers, Opt. Express 14, 1658 (2006).

[8] A. Lavrinenko, A. Tetu, L. H. Frandsen, and P. I. Borel, in CLEO/QELS Technical Digest (Optical Society of America, Washington, DC, 2006), p. QFC6.

[9] H. Altug and J. Vuckovic, Appl. Phys. Lett. 86, 111102 (2005).

[10] D. N. Christodoulides, F. Lederer, and Y. Silberberg, Nature (London) 424, 817 (2003).

[11] P. Millar, R. M. De la Rue, T.F. Krauss, J. S. Aitchison, N. G. R. Broderick, and D. J. Richardson, Opt. Lett. 24, 685 (1999).

[12] G.P. Agrawal, Nonlinear Fiber Optics (Academic, New York, 1988).

[13] W. C. K. Mak, P. L. Chu, and B. A. Malomed, J. Opt. Soc. Am. B 15, 1685 (1998).

[14] W. C. K. Mak, B. A. Malomed, and P. L. Chu, Phys. Rev. E 69, 066610 (2004).

[15] A. Gubeskys and B. A. Malomed, Eur. Phys. J. D 28, 283 (2004).

[16] T. Dohnal and A. B. Aceves, Stud. Appl. Math. 115, 209 (2005).

[17] Y. Silberberg, Opt. Lett. 15, 1282 (1990).

[18] H. S. Eisenberg, R. Morandotti, Y. Silberberg, S. Bar Ad, D. Ross, and J. S. Aitchison, Phys. Rev. Lett. 87, 043902 (2001).

[19] B. A. Malomed, D. Mihalache, F. Wise, and L. Torner, J. Opt. B 7, R53 (2005).

[20] S. Droulias, K. Hizanidis, J. Meier, and D. N. Christodoulides, Opt. Express 13, 1827 (2005). 